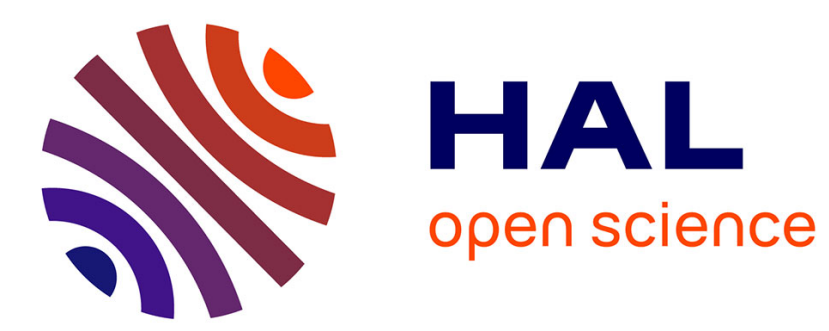

\title{
Mesure du taux de polarisation rectiligne des différentes raies d'une source Mössbauer
}

\author{
M. Henry, F. Varret
}

\section{To cite this version:}

M. Henry, F. Varret. Mesure du taux de polarisation rectiligne des différentes raies d'une source Mössbauer. Revue de Physique Appliquée, 1979, 14 (3), pp.509-515. 10.1051/rphysap:01979001403050900 . jpa-00244621

\section{HAL Id: jpa-00244621 \\ https://hal.science/jpa-00244621}

Submitted on 1 Jan 1979

HAL is a multi-disciplinary open access archive for the deposit and dissemination of scientific research documents, whether they are published or not. The documents may come from teaching and research institutions in France or abroad, or from public or private research centers.
L'archive ouverte pluridisciplinaire HAL, est destinée au dépôt et à la diffusion de documents scientifiques de niveau recherche, publiés ou non, émanant des établissements d'enseignement et de recherche français ou étrangers, des laboratoires publics ou privés. 


\title{
Mesure du taux de polarisation rectiligne des différentes raies d'une source Mössbauer
}

\author{
M. Henry et F. Varret \\ E.R.A. CNRS n ${ }^{\circ} 682$ Groupe de Physique et Chimie du Solide, \\ Faculté des Sciences du Mans, F 72017 Le Mans Cedex, France
}

(Reçu le 27 octobre 1978, accepté le ler décembre 1978)

\begin{abstract}
Résumé. - Nous avons testé une source Mössbauer polarisée rectilignement, réalisée sous la forme d'une source commerciale de ${ }^{57} \mathrm{Co}$ dans du fer métallique, soumise à un champ magnétique transversal de $0,4 \mathrm{~T}$. Les spectres obtenus avec des absorbeurs polarisés, soit de fer aimanté, soit de fluosilicate ferreux monocristallin, orientés parallèlement, puis perpendiculairement à l'aimantation de la source, permettent de mesurer le taux de polarisation rectiligne des différentes raies de la source. L'interprétation quantitative des intensités de raies tient compte des effets d'épaisseur, auto-absorption et absorption, inhérents à l'expérience.
\end{abstract}

\begin{abstract}
We have investigated a linearly polarized Mössbauer source, made of a standard ${ }^{57} \mathrm{Co}: \mathrm{Fe}$ metal source submitted to a transverse $0.4 \mathrm{~T}$ field. The linear polarization rates of the different lines have been deduced from the spectra of polarized absorbers, made of either magnetized iron, or ferrous fluosilicate single crystal, oriented either parallel or perpendicular to the source magnetization. The quantitative interpretation of the line intensities takes into account thickness effects involved in the experiment : self absorption and absorption.
\end{abstract}

1. Introduction. - Une méthode classique pour obtenir une source Mössbauer polarisée rectilignement consiste à aimanter à saturation une source de ${ }^{57} \mathrm{Co}$ dans du fer métallique [1], la direction de l'aimantation étant perpendiculaire à la direction de propagation du rayonnement (Fig. 1). En pratique, la saturation magnétique et donc la polarisation totale sont difficiles à réaliser parfaitement; notre étude récente sur les effets d'auto-absorption dans une source polarisée d'origine commerciale [2] permettait de prévoir que la polarisation ne serait pas totale et que les diverses raies du spectre pouvaient présenter des taux de polarisation différents.

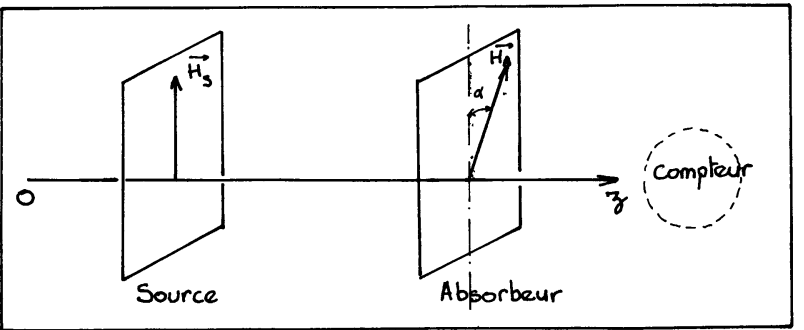

Fig. 1. - Schéma de principe des manipulations.

[Experimental set.]
Le dispositif utilisé, analogue aux dispositifs de polarimétrie conventionnelle, est représenté sur la figure 1. L'analyseur (en position d'absorbeur) est d'abord une feuille de fer métallique aimantée à saturation (vu sa forme et sa perméabilité) par un champ de $0,4 \mathrm{~T}$. Deux expériences, en champs parallèles $(\alpha=0)$ ou perpendiculaires $\left(\alpha=90^{\circ}\right)$, ont été réalisées (Fig. 2).

Par ailleurs, les effets d'épaisseur dans la source (auto-absorption) comme dans l'absorbeur (absorption) ont été étudiés préalablement [2.3].

\section{Dispositif expérimental. -2.1 DesCription dU} MATÉRIEL. - La source utilisée (Amersham, ref. CTD 14, activité $10 \mathrm{mCi}$ de ${ }^{57} \mathrm{Co}$ dans du fer) est placée dans un champ magnétique de $0,4 \mathrm{~T}$.

L'absorbeur utilisé (Amersham, épaisseur 12,5 $\mu$, fer naturel) est placé dans un champ identique.

Dans le cas de la disposition $\mathbf{H}_{\mathrm{S}}$ parallèle à $\mathbf{H}_{\mathrm{A}}$ : la source et l'absorbeur sont en fait placés dans l'entrefer d'un seul aimant permanent; dans la disposition $\mathbf{H}_{\mathrm{S}}$ perpendiculaire à $\mathbf{H}_{\mathrm{A}}$ nous utilisons deux aimants permanents identiques. Dans les deux cas la direction du (ou des) champ(s) magnétique(s) est perpendiculaire à la direction d'observation. 


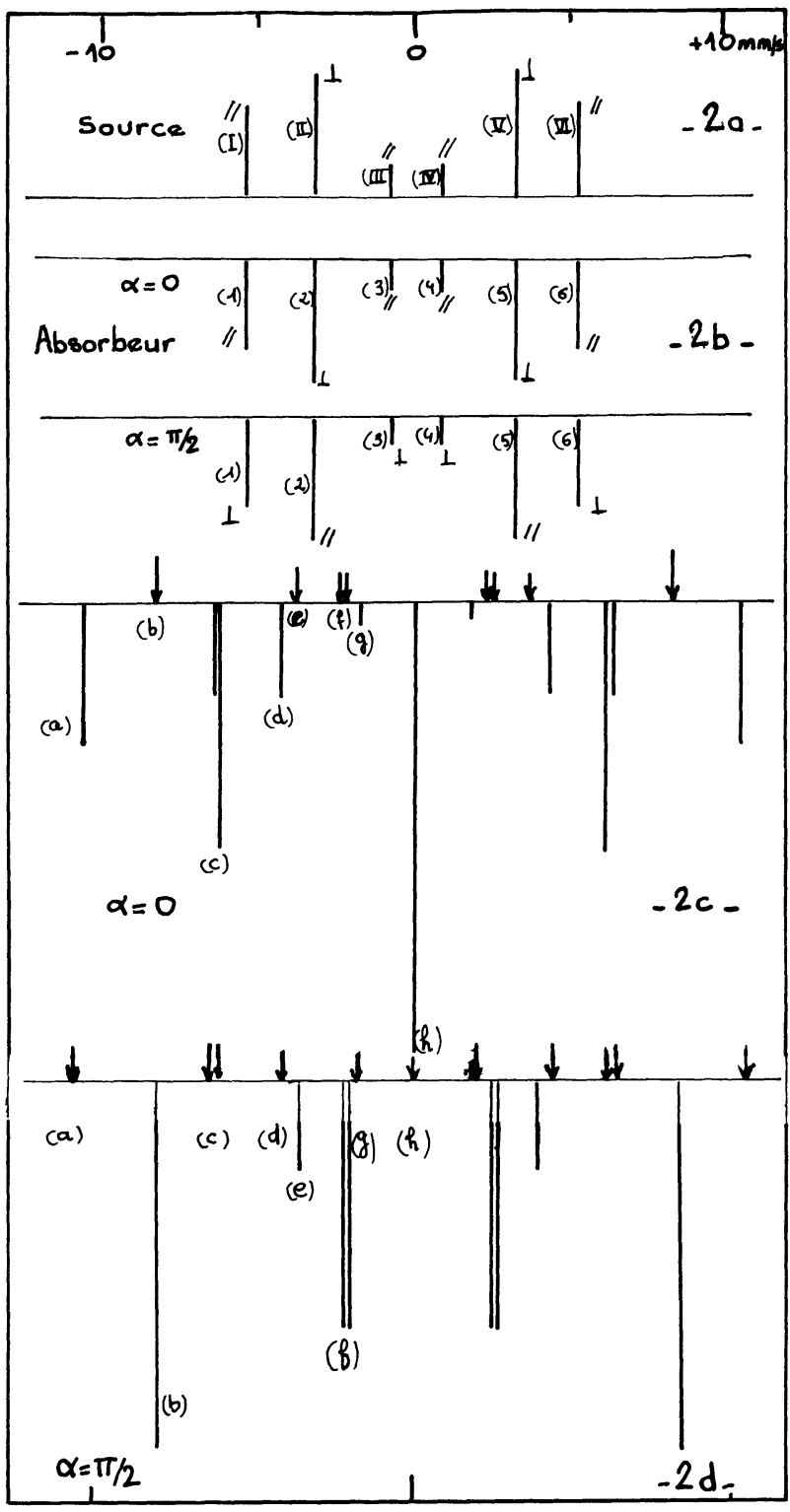

Fig. 2. - Spectres théoriques dans le cas idéal : source (a), absorbeur (b), expériences source-absorbeur $\alpha=0$ (c), $90^{\circ}$ (d). L'état de polarisation des raies $(/ /$ ou $\perp)$ est repéré par rapport à la direction du champ appliqué sur la source.

[Predicted spectra (ideal case) : source (a), absorber (b), source : absorber experiment $\alpha=0$ (c), $\alpha=90^{\circ}$ (d). The polarization of lines $(/ /$ or $\perp)$ refers to the direction of the applied field on the source.]

L'ouverture maximum du faisceau dans les expériences que nous présentons n'a pas dépassé $10^{\circ}$ (ce qui a nécessité l'usage d'un diaphragme pour l'expérience en champs parallèles). Un spectre obtenu sans diaphragme dans le cas parallèle (ouverture $\sim 30^{\circ}$ ) ayant donné des résultats identiques, il nous a paru légitime de négliger les effets de l'inclinaison possible du rayonnement par rapport à l'axe de l'expérience.

2.2 DÉPOUILLEMENT DES SPECTRES EXPÉRIMENTAUX. - Les spectres obtenus sont reportés figure 3. Les résultats en seront analysés dans la partie 3 .

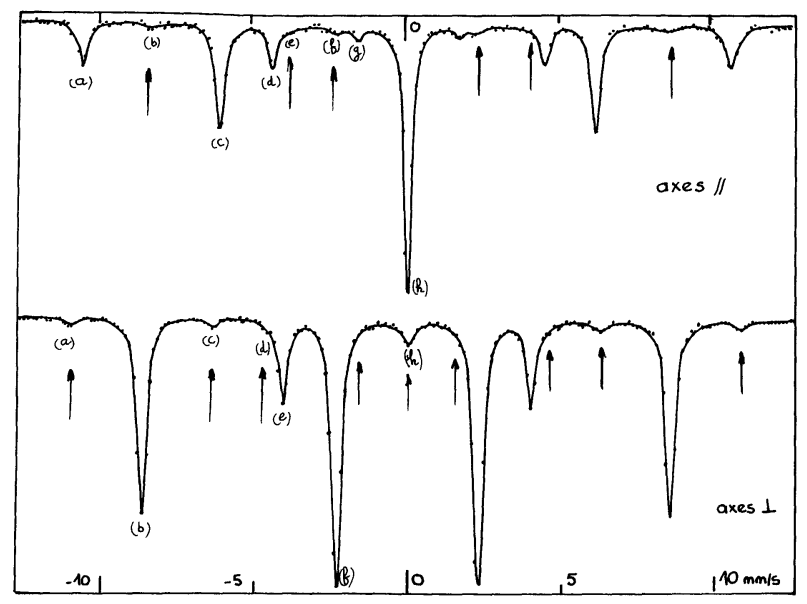

Fig. 3. - Spectres expérimentaux (en trait plein : spectre ajusté) : absorber de fer aimanté les $\downarrow$ indiquent les raies interdites.

[Experimental spectra (full line : fitted spectra) absorber : magnetized iron foil arrows show the forbidden lines positions.]

Les intensités mesurées expérimentalement sont proportionnelles aux surfaces des raies, supposées lorentziennes. L'ajustement des spectres a été fait de la manière suivante, compte tenu de la faible intensité de certaines raies :

- Dans le cas de l'orientation parallèle, le spectre expérimental a été ajusté sur un spectre composé de 7 doublets symétriques et d'une raie centrale.

- Dans le cas de l'orientation perpendiculaire l'ajustement a été fait avec 6 doublets symétriques et une raie centrale.

Pour certaines raies faibles, la largeur de raie était un paramètre contraint [4] à rester égal à la largeur d'une raie intense d'origine comparable.

Les largeurs mesurées varient entre 0,19 et $0,15 \mathrm{~mm} / \mathrm{s}$ ce qui donne un ordre de grandeur de l'incertitude relative introduite par cette contrainte sur l'intensité des raies faibles (incertitude $\sim<12 \%$ ).

Nous rappelons par ailleurs la valeur des intensités relatives des raies d'émission de la source et de l'absorbeur, mesurées antérieurement $[2,3]$ :

- pour l'absorbeur :

$$
\begin{aligned}
& Q_{1}=Q_{6}=19,0 \% ; \quad Q_{2}=Q_{5}=23,0 \% ; \\
& Q_{3}=Q_{4}=8,0 \%
\end{aligned}
$$

- pour la source :

$$
\begin{aligned}
& Q_{\mathrm{I}}=Q_{\mathrm{VI}}=19,7 \% ; \quad Q_{\mathrm{II}}=Q_{\mathrm{V}}=22,5 \% ; \\
& Q_{\mathrm{III}}=Q_{\mathrm{IV}}=7,8 \% .
\end{aligned}
$$

3. Calcul des intensités des raies. - Nous supposons que l'absorbeur de fer est totalement polarisé sous l'effet du champ appliqué $0,4 \mathrm{~T}$ (ce qui paraît raisonnable vu sa forme qui annule le champ démagnétisant 
et sa forte perméabilité garantie par le recuit et la pureté du matériau; avec un matériau analogue Imbert [1a] a observé que quelques centaines d'œrsteds étaient suffisants pour produire la saturation magnétique). Les polarisations rectilignes parallèle et perpendiculaire à $H_{\mathrm{A}}$ sont les états de base dans la présente interprétation.

Nous supposons que la source est dans un état de polarisation défini comme suit : les raies I, III, IV, VI correspondant à des transitions $\Delta m= \pm 1$ pures sont affectées d'un même taux $t$ de polarisation recti- ligne ; les raies II et $\mathrm{V}(\Delta m=0)$ du taux $t^{\prime}$. Dans un spectre quelconque, pour lequel les raies ne correspondraient pas à des transitions pures $\Delta m=1,0,-1$, il faudrait associer à chaque raie une polarisation particulière.

Les intensités relatives des composantes polarisées de la source sont indiquées tableau I.

Ceci permet d'établir les expressions des intensités relatives des raies du spectre source fer-absorbeur fer en fonction de $t$ et $t^{\prime}$ qui sont indiquées dans le tableau I.

Il est intéressant de calculer, pour chaque orien-

Tableau I. - Intensités relatives des raies (calculées dans le cas d'un absorbeur magnétique). Les indices I... VI sont relatifs à la source, $1 \ldots 6$ à l'absorbeur et $\mathrm{a} . . . \mathrm{h}$ au spectre résultant. Les signes \pm sont relatifs aux cas parallèle et perpendiculaire respectivement.

La direction de référence est le champ appliqué à la source. P et I indiquent le caractère permis ou interdit de la transition (dans le cas idéal totalement polarisé).

[Relative intensities of the Mössbauer lines (calculated) for a magnetic absorber. Source labels are I... VI, absorber labels are $1 \ldots 6$; for the source-absorber experiment, labels are a... h.

The signs \pm respectively refer to parallel and perpendicular directions for $H_{\mathrm{A}}$ and $H_{\mathrm{s}}$. The reference direction is that of $H_{\mathrm{S}} . P$ and $I$ refer to the allowed or forbidden character of the transition in the ideal case.]

\section{Raies}

I. VI

II, V

III. IV

3.4

(I, 3) (III, 1)

$(\mathrm{I}, 4)(\mathrm{IV}, 1)$

(II. 3) (III. 2)

$(\mathrm{I}, 5)(\mathrm{V}, 1)$

(II, 4) $(\mathrm{IV}, 2)\}$

(III. 3)

(I, 6) (II. 5)
Intensités relatives

$$
\begin{aligned}
& Q_{\mathrm{I}}=Q_{\mathrm{I}}^{\mathrm{II}}+Q_{\mathrm{I}}^{\perp}=Q_{\mathrm{I}}\left(\frac{1+\bar{t}}{2}\right)+Q_{\mathrm{I}}\left(\frac{1-t}{2}\right) \\
& Q_{\mathrm{II}}=Q_{\mathrm{II}}+Q_{\mathrm{II}}^{\perp}=Q_{\mathrm{II}}\left(\frac{1-t^{\prime}}{2}\right)+Q_{\mathrm{II}}\left(\frac{1+t^{\prime}}{2}\right) \\
& Q_{\mathrm{III}}=Q_{\mathrm{III}}+Q_{\mathrm{III}}^{\perp}=Q_{\mathrm{III}}\left(\frac{1+t}{2}\right)+Q_{\mathrm{III}}\left(\frac{1-t}{2}\right)
\end{aligned}
$$

(III. 4)$$
Q_{1}=Q_{1}^{\|}\left(Q_{1}^{\perp}\right)
$$$$
Q_{2}=Q_{2}^{\perp}\left(Q_{2}^{\|}\right)
$$$$
Q_{3}=Q_{3}^{\|}\left(Q_{3}^{\perp}\right)
$$

$$
Q_{\mathrm{a}}=\left(\frac{1 \pm t}{2}\right) Q_{\mathrm{I}} Q_{1}
$$

$$
\begin{gathered}
Q_{\mathrm{I}}+Q_{\mathrm{II}}+Q_{\mathrm{III}}=0,5 \\
Q_{1}+Q_{2}+Q_{3}=0,5 \\
\text { raies entièrement } \\
\text { polarisées }
\end{gathered}
$$

$$
H_{\mathrm{A}} / / H_{\mathrm{S}} \quad H_{\mathrm{A}} \perp H_{\mathrm{S}}
$$

P

$$
Q_{\mathrm{d}}=\frac{1 \pm t}{2}\left(Q_{\mathrm{I}} Q_{3}+Q_{\mathrm{III}} Q_{1}\right)
$$

$$
Q_{\mathrm{e}}=\frac{1 \mp t}{2} Q_{\mathrm{III}} Q_{2}+Q_{\mathrm{II}} Q_{3} \frac{1 \mp t^{\prime}}{2}
$$


tation, la somme des intensités des raies permises, $P$, ou interdites, $I$ :

$$
\begin{aligned}
& \begin{array}{l}
(1 \pm t)\left(Q_{1}+Q_{3}\right)\left(Q_{\mathrm{I}}+Q_{\mathrm{III}}\right)+ \\
+(1 \pm t) Q_{2} Q_{\mathrm{II}}=P / 2 \quad(\text { ou } I / 2) \\
(1 \pm t) Q_{2}\left(Q_{\mathrm{I}}+Q_{\mathrm{III}}\right)+ \\
+\left(1 \pm t^{\prime}\right)\left(Q_{1}+Q_{3}\right) Q_{\mathrm{II}}=I / 2 \quad(\text { ou } P / 2)
\end{array}
\end{aligned}
$$

$(+$ ou,$- P$ ou $I$ selon que les deux champs magnétiques sont parallèles ou perpendiculaires respectivement) avec

$$
\begin{aligned}
& Q_{1}+Q_{2}+Q_{3}=0,5 \\
& Q_{\mathrm{I}}+Q_{\mathrm{II}}+Q_{\mathrm{III}}=0,5 .
\end{aligned}
$$

Si l'on remarque préalablement que

$$
Q_{1(\mathrm{I})}+Q_{3(\mathrm{III})} \simeq Q_{2(\mathrm{II})},
$$

on voit facilement que le rapport $I / P$ doit avoir sensiblement la même valeur dans les deux expériences, ce qui est effectivement observé avec une bonne précision $(5,3 \%$ en $/ /$ et $6,0 \% \perp)$. Dans ces conditions, la seule donnée du rapport $I / P$ ne suffit évidemment pas à déterminer simultanément $t$ et $t^{\prime}$.

Par contre on peut aisément déterminer un taux moyen de polarisation défini par $t_{\mathrm{m}}=\left(t+t^{\prime}\right) / 2$. Compte tenu du fait que $Q_{\mathrm{I}(1)}+Q_{\mathrm{III}(3)} \simeq Q_{\mathrm{II}(2)}$ l'expression de $I / P$ peut se transformer pour donner de manière approchée :

$$
\frac{1-t_{\mathrm{m}}}{1+t_{\mathrm{m}}} \simeq \frac{I}{P}
$$

d'où nous déduisons :

$$
t_{\mathrm{m}}=(89 \pm 1) \%
$$

Partant, en reprenant les expressions des raies en fonction de $t$ et $t^{\prime}$ (cf. tableau I) nous déterminons $t$. En effet, la raie (a) dans le cas perpendiculaire, qui est bien résolue et assez loin de toute autre, a une intensité qui ne dépend que de $t$; la raie (f) a une intensité qui s'écrit en fonction de $t_{\mathrm{m}}$ :

$$
Q_{\mathrm{f}}=\left(1+t_{\mathrm{m}}\right)\left\{Q_{2}\left(Q_{\mathrm{I}}+Q_{\mathrm{III}}\right)+Q_{\mathrm{II}}\left(Q_{1}+Q_{3}\right)\right\} \text {. }
$$

En comparant $Q_{\mathrm{a}}$ et $Q_{\mathrm{f}}$ on en déduit une valeur de $t$ indépendante de la valeur $t_{\mathrm{m}}$, soit $t \sim 81 \%$, d'où l'on déduit $t^{\prime} \sim 97 \%$.

L'analyse du groupe de raies (c), qui dépend de $t^{\prime}$ plus que de $t$ permet une autre mesure de $t^{\prime}$, un peu moins précise : $t^{\prime}=91 \%, t=88 \%$. Compte tenu des précisions, nous pouvons adopter :

$$
t \simeq 84 \%, \quad t^{\prime} \simeq 94 \%( \pm 3 \%)
$$

4. Expérience avec un absorbeur de fluosilicate ferreux monocristallin. - L'absorbeur·est une lame mince de fluosilicate ferreux (épaisseur $=0,45 \mathrm{~mm}$ ) dont l'axe rhomboédrique est placé perpendiculairement à la direction du rayonnement.

Le spectre résultant de l'étude, avec la source polarisée, de l'absorbeur fluosilicate dépend de l'angle $\alpha$ entre la direction du champ interne de la source et l'axe du gradient de champ du monocristal (Fig. 4).

Les spectres obtenus sont reportés figure 5. L'ajustement a été fait sur les mêmes bases que celles indiquées en 2.2 avec cependant une différence due au fait qu'ici les spectres résultants ne sont plus symétriques par rapport au zéro d'énergie. Ceci nous a conduit à faire des ajustements par raies séparées plutôt que par doublets. Dans le cas de l'orientation parallèle $(\alpha=0)$ l'ajustement a été fait avec un spectre composé de 4 doublets et de 4 raies simples (dans le cas perpendiculaire : 3 doublets et 6 raies simples).

Tableau II. - Intensités relatives des raies Mössbauer : valeurs idéales (polarisation totale, pas d'effets d'épaisseur), expérimentales, calculées avec les valeurs $t=84 \%, t^{\prime}=94 \%$ dans le cas de l'absorbeur de fer.

[Values of the relative intensities of the Mössbauer lines : ideal (total polarization, without thickness effects), experimental. calculated $\left(t=84 \%, t^{\prime}=94 \%\right)$.]

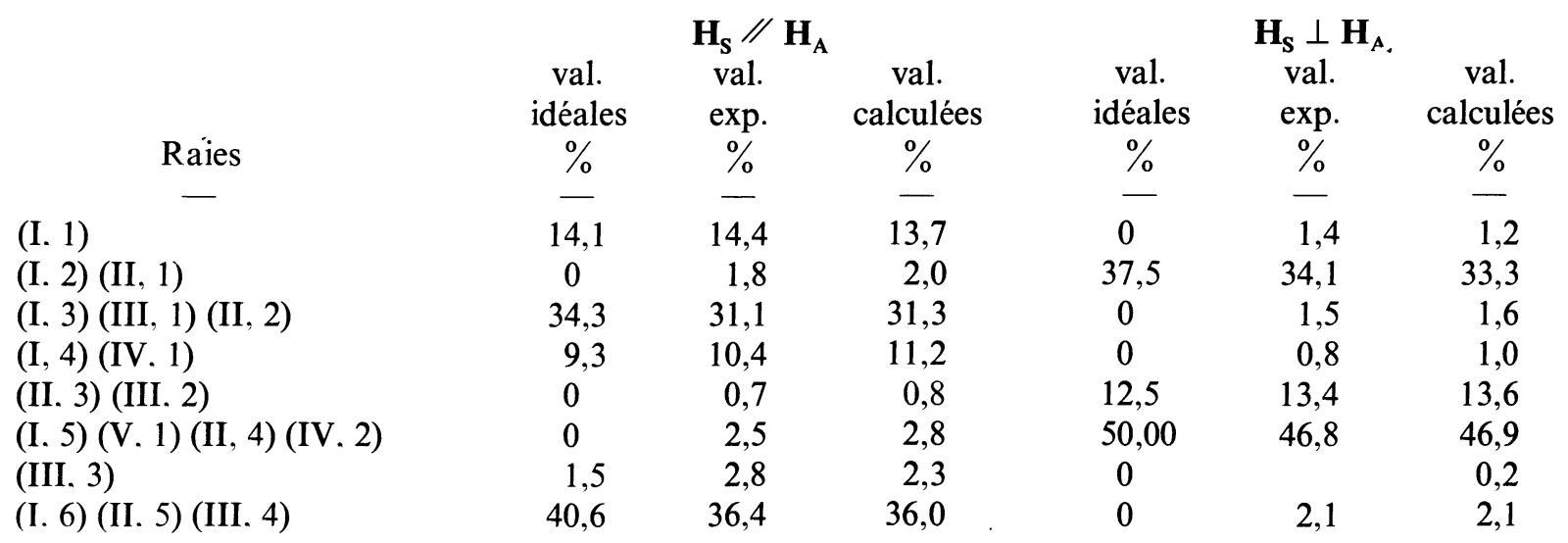




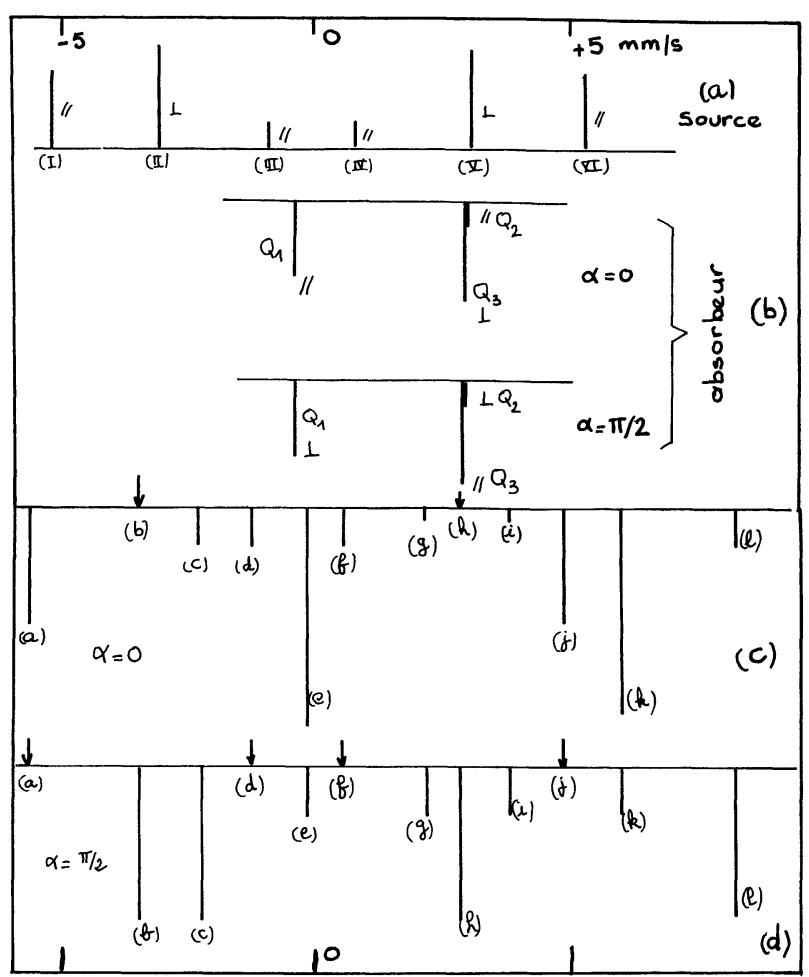

Fig. 4. - Spectres théoriques dans le cas idéal : source (a)-absorbeur (b), expériences source-absorbeur $\alpha=0$ (c), 90 (d). L'état de polarisation des raies est repéré par rapport à la direction de champ appliqué sur la source.

[Predicted spectra (ideal case) : source (a), absorber (b). Source absorber experiments $\alpha=0$ (c), $\alpha=90^{\circ}$ (d). The polarization lines refers to the direction of the applied field on the source.]

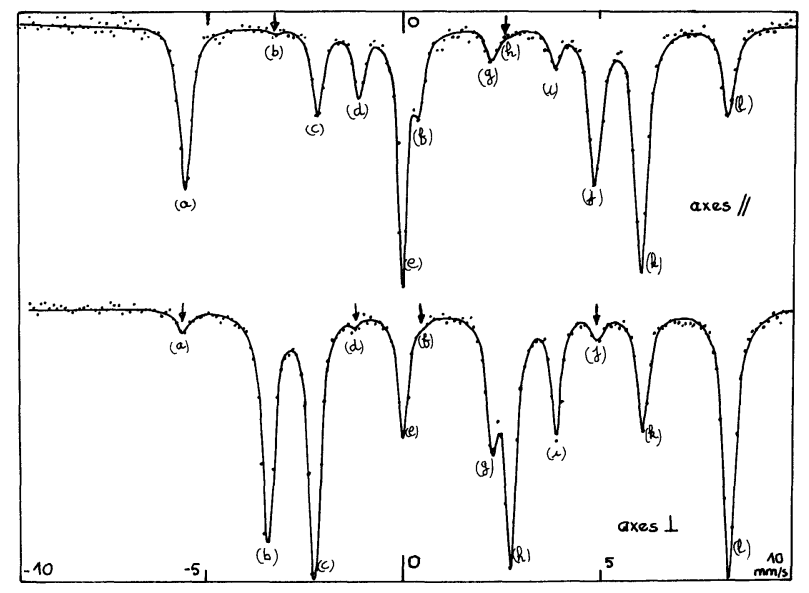

Fig. 5. - Spectres expérimentaux : absorbeur fluosilicate ferreux, les flèches indiquent les raies interdites.

[Experimental spectra-using a ferrous fluosilicate absorber, arrows show the forbidden lines positions.]

Comme en 2.2 (et pour les mêmes raisons) certaines contraintes ont été introduites sur les largeurs de raies.

De la même manière que précédemment nous avons calculé les intensités relatives des raies du spectre source fer-absorbeur fluosilicate ferreux en fonction de $t$ et $t^{\prime}$. Les expressions sont rassemblées dans le tableau III. On constate qu'ici (contrairement aux expériences source fer-absorbeur fer) il n'y a pas symétrie entre ce qui est permis et interdit pour les cas $\alpha=0$ et $\alpha=\pi / 2$ : ceci est dû au fait que les raies d'intensités relatives $Q_{2}$ et $Q_{3}$ de l'absorbeur coïncident en énergie avec des polarisations différentes.

Il y a donc deux raies théoriquement interdites $\left(Q_{\mathrm{b}}, Q_{\mathrm{h}}\right)$ pour l'orientation parallèle et quatre pour l'orientation perpendiculaire $\left(Q_{\mathrm{a}}, Q_{\mathrm{j}}, Q_{\mathrm{d}}, Q_{\mathrm{f}}\right)$. A partir des 2 spectres $(\alpha=0, \alpha=\pi / 2)$, cf. tableau IV, on déduit le rapport

$$
\frac{1-t^{\prime}}{1-t}=\frac{Q_{\mathrm{III}}+Q_{\mathrm{I}}}{Q_{\mathrm{II}}} \frac{Q_{\mathrm{b}}^{(1)}}{Q_{\mathrm{a}}^{(2)}+Q_{\mathrm{d}}^{(2)}}=0,35
$$

(1) et (2) correspondant respectivement aux mesures dans le cas parallèle et perpendiculaire.

Ce rapport correspond bien à ce que l'on a trouvé précédemment. Par ailleurs, on peut trouver

$$
t_{\mathrm{m}}=(87 \pm 1) \%
$$

ce qui donne finalement $t=81 \%$ et $t^{\prime}=93 \%$ en assez bon accord avec les valeurs obtenues à l'aide de l'analyseur de fer.

Compte tenu de ces résultats, en utilisant les expressions indiquées tableau III, èt connaissant la valeur de $Q_{1}$ mesurée sur un spectre conventionnel, nous obtenons finalement :

$$
Q_{1}=38,0 \%, \quad Q_{2}=14,8 \%, \quad Q_{3}=47,2 \% \text {. }
$$

Pour tester globalement l'interprétation, nous comparons les intensités théoriques des raies déduites des valeurs précédentes de $t, t^{\prime}, Q_{1}, Q_{2}, Q_{3}$, aux valeurs mesurées (tableau IV). L'accord est moins bon qu'avec l'analyseur de fer (probablement en raison des difficultés théoriques et pratiques liées à la mesure des surfaces de raies présentant un certain recouvrement).

Les valeurs de $Q_{1}, Q_{2}, Q_{3}$ obtenues présentent une certaine vraisemblance. Une interprétation analogue à celle de [3] permet de leur faire correspondre une épaisseur effective $T=3,0$ conduisant à la valeur $f \sim 0,5$. Cette valeur ne diffère pas trop de la valeur $f=0,3$ obtenue par des mesures précises de biréfringence [1a] (compte tenu du fait que c'est l'écart entre les valeurs idéales et expérimentales de $Q_{\mathrm{i}}$ qui est à la base de la présente évaluation de $f$ ).

\section{Discussion : orientation des spins dans la source. -} L'introduction d'un taux de $t^{\prime}$ de polarisation différent de 1 pour les raies II et $\mathbf{V}$ de la source conduit à affiner l'hypothèse rendant compte de la polarisation incomplète de la source [2]. On avait supposé que les axes de quantification des noyaux émetteur et absorbeur de la source faisaient un angle $(\Pi / 2-\delta)$ avec la direction d'observation et que ces axes restaient parallèles entre eux (effet $\mathrm{n}^{\mathrm{o}} 1$, inclinaison longitudinale des spins : figure 6). La nécessité d'introduire 
Tableau III. - Intensités relatives des raies (calculées dans le cas de l'absorbeur quadrupolaire) les indices a... 1 sont relatifs au spectre résultant. Les signes \pm sont relatifs aux cas parallèle et perpendiculaire respectivement.

[Relative intensities of the Mössbauer lines (calculated) lables a... 1 are for the source-absorbeur (ferrous fluosilicate) experiment. The signs \pm refer to axes oriented parallel or perpendicular respectively.]

Raies

I-VI

II-V

III-IV

(I-1) et (VI-1)

(II-1) et (V-1)

(I-2) (I-3) et (VI-2) (VI-3)

(III-1) et (IV-1)

(II-2) (II-3) et (V-2) (V-3)

(III-2) (III-3) et (IV-2) (IV-3)
Intensités relatives

$$
\begin{aligned}
& Q_{\mathrm{I}}=Q_{\mathrm{I}}^{\|}+Q_{\mathrm{I}}^{\perp}=Q_{\mathrm{I}}\left(\frac{1+t}{2}\right)+Q_{\mathrm{I}}\left(\frac{1-t}{2}\right) \\
& Q_{\mathrm{II}}=Q_{\mathrm{II}}^{\|}+Q_{\mathrm{II}}^{\perp}=Q_{\mathrm{II}}\left(\frac{1-t^{\prime}}{2}\right)+Q_{\mathrm{II}}\left(\frac{1+t^{\prime}}{2}\right) \\
& Q_{\mathrm{III}}=Q_{\mathrm{III}}^{\|}+Q_{\mathrm{III}}^{\perp}=Q_{\mathrm{III}}\left(\frac{1+t}{2}\right)+Q_{\mathrm{III}}\left(\frac{1-t}{2}\right) \\
& Q_{1}=Q_{1}^{\|}\left(Q_{1}^{\perp}\right) \\
& Q_{2}+Q_{3}=Q_{2}^{\|}+Q_{3}^{\perp}\left(Q_{2}^{\perp}+Q_{3}^{\|}\right)
\end{aligned}
$$

$$
Q_{\mathrm{I}}+Q_{\mathrm{II}}+Q_{\mathrm{III}}=0,5
$$$$
Q_{1}+Q_{2}+Q_{3}=1
$$

Remarques$$
+
$$$$
Q_{\mathrm{a}}=Q_{\mathrm{I}} Q_{1}\left(\frac{1 \pm t}{2}\right)=Q_{\mathrm{j}}
$$$$
\stackrel{\perp}{-}
$$$$
\text { P I }
$$$$
\text { I } \quad \mathbf{P}
$$$$
Q_{\mathrm{b}}=Q_{\mathrm{II}} Q_{1}\left(\frac{1 \mp t^{\prime}}{2}\right)=Q_{\mathrm{h}}
$$$$
Q_{\mathrm{c}}=Q_{\mathrm{1}}\left[Q_{2}\left(\frac{1 \pm t}{2}\right)+Q_{3}\left(\frac{1 \mp t}{2}\right)\right]=Q_{1}
$$$$
\mathbf{P} \quad \mathbf{P}
$$$$
\text { P I }
$$$$
Q_{\mathrm{d}}=Q_{\mathrm{III}} Q_{1}\left(\frac{1 \pm t}{2}\right)=Q_{\mathrm{f}}
$$$$
Q_{\mathrm{e}}=Q_{\mathrm{II}}\left[Q_{2}\left(\frac{1 \mp t^{\prime}}{2}\right)+Q_{3}\left(\frac{1 \pm t^{\prime}}{2}\right)\right]=Q_{\mathrm{k}}
$$$$
\text { P P }
$$$$
Q_{\mathrm{g}}=Q_{\mathrm{III}}\left[Q_{2}\left(\frac{1 \pm t}{2}\right)+Q_{3}\left(\frac{1 \mp t}{2}\right)\right]=Q_{\mathrm{i}}
$$

P P
Tableau IV. - Intensités relatives des raies Mössbauer : valeurs expérimentales et valeurs calculées, dans le cas de l'analyseur de fluosilicate ferreux.

[Experimental and calculated values of the relative intensities of the Mössbauer lines, in the case of a ferrous fluosilicate analyzer.]

\begin{tabular}{ccccc} 
& \multicolumn{2}{c}{ Orientation $/ /$} & \multicolumn{2}{c}{ Orientation $\perp$} \\
Raies & $\begin{array}{c}\text { expéri- } \\
\text { mentales }\end{array}$ & calculées & $\begin{array}{c}\text { expéri- } \\
\text { mentales }\end{array}$ & calculées \\
- & - & - & - & - \\
$Q_{\mathrm{a}}+Q_{\mathrm{j}}$ & 28,9 & 27,1 & 3,2 & 2,8 \\
$Q_{\mathrm{b}}+Q_{\mathrm{h}}$ & 1,2 & 1,2 & 32,8 & 33,1 \\
$Q_{\mathrm{c}}+Q_{1}$ & 14,3 & 14,1 & 35,1 & 34,8 \\
$Q_{\mathrm{d}}+Q_{\mathrm{f}}$ & 9,4 & 10,7 & 1,1 & 1,1 \\
$Q_{\mathrm{e}}+Q_{\mathrm{k}}$ & 40,6 & 41,4 & 14,2 & 14,4 \\
$Q_{\mathrm{g}}+Q_{\mathrm{i}}$ & 5,6 & 5,6 & 13,6 & 13,8
\end{tabular}

$t^{\prime} \neq .1$ pour rendre compte des spectres expérimentaux conduit à envisager un deuxième effet caractérisé par une déviation transversale moyenne entre deux noyaux émetteurs (effet $\mathrm{n}^{\circ} 2$, Fig. 6).

$\mathrm{Si}$ nous supposons que les deux effets agissent indépendamment sur les taux de polarisation, nous constatons d'abord que la différence $\left(t^{\prime}-t\right)$ due à

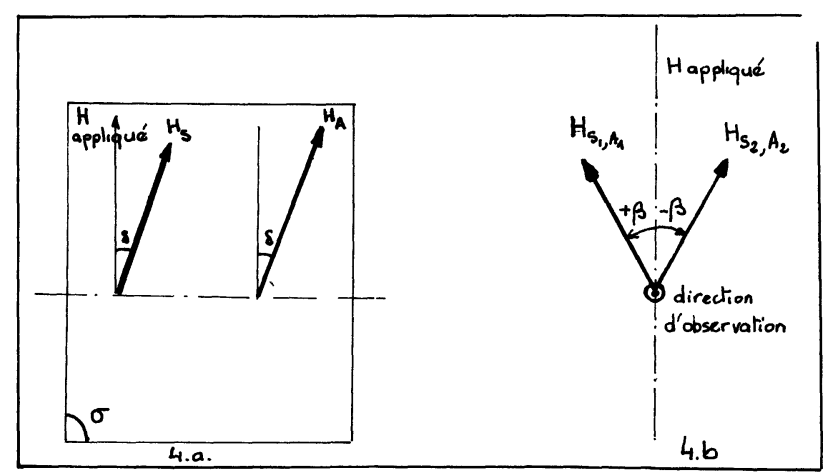

Fig. 6. - Hypothèse de polarisation incomplète : effet $\mathrm{n}^{0} 1$ (inclinaison longitudinale des spins [2]); effet $\mathrm{n}^{\circ} 2$ (dispersion transversale des spins).

[Partial polarization assumptions : spins $\left\{\begin{array}{l}\text { effect } n^{\circ} 1 \text { (longitudinal tilting of the spins) } \\ \text { effect } n^{\circ} 2 \text { (transversal tilting of the spins). }\end{array}\right.$

l'effet 1 est comparable à celle qui était prévue par l'analyse des effets d'auto-absorption [2] (respectivement $10 \%$ et $7 \%$ ).

Il est intéressant aussi d'avoir un ordre de grandeur de l'écart transversal moyen $2 \beta$. Considérons deux 
noyaux émetteurs déviés de $\pm \beta$ par rapport à la direction du champ appliqué. Le taux de polarisation $t^{\prime}$, qui n'est affecté que par l'effet $\mathrm{n}^{\circ} 2$, se calcule aisément : $t^{\prime}=\cos ^{2} \beta-\sin ^{2} \beta$. La valeur $t^{\prime}=94 \%$ conduit à une déviation $\beta \sim 10^{\circ}$; cette dernière valeur très proche de la déviation longitudinale $\delta \sim 11^{\circ}[2]$ suggère un modèle de domaines ferromagnétiques juxtaposés dont la direction d'aimantation présenterait par rapport au champ magnétique une distribution angulaire sensiblement axiale; l'écart moyen entre l'aimantation des domaines et le champ appliqué serait de l'ordre de $10^{\circ}$.

6. Conclusions. - Nous avons obtenu un bon accord quantitatif entre les valeurs mesurées et calculées de toutes les raies de nos quatre expériences de polarisation. Cet accord permet d'avoir confiance dans les valeurs mesurées de $t$ et $t^{\prime}$, et en particulier dans le signe de leur différence $\left(t^{\prime}>t\right)$. La méthode exposée nous paraît utilisable en routine pour mesurer les taux de polarisation de raies d'émission Mössbauer. Il est à remarquer qu'une analyse plus rustique négligeant les divers effets d'épaisseur conduirait à des valeurs sensiblement identiques pour $t$ et $t^{\prime}$.

En ce qui concerne la source commerciale que nous avons ainsi testée, l'effet de dispersion de la direction des spins, précédemment décelé dans un plan longitudinal se trouve également mis en évidence dans un plan transversal; cela traduit en fait une dispersion axiale des spins. Une source dont le traitement métallurgique pourrait être identique à celui de l'absorbeur, verrait bien sûr disparaître cette dispersion des spins et présenterait sur toutes ses raies une polarisation complète.

\section{Bibliographie}

[1] (a) IMBERT, Thèse CEN, Saclay 1965 et $J$. Physique 27 (1966) 429.

(b) Frauenfelder, H., Nagle, G. E., Taylor, R. D., Cochran D. R. F., Visscher, W. M., Phys. Rev. 126 (1962) 1065.
[2] Henry, M., Varret, F., à paraître dans la Revue Phys. Appl. [3] Henry, M., Varret, F., soumis au Journal de Physique.

[4] Teillet, J., Varret, F., Programme d'ajustement des spectres (non publié). 